\title{
The Current Status of Bacillus Calmette-Guérin Vaccine Protective Efficacy
}

\author{
Méndez-Samperio Patricia
}

Departamento de Inmunología, Escuela Nacional de Ciencias Biológicas, IPN. Prol. Carpio y Plan de Ayala. CD México 11340

México.

\begin{tabular}{ll}
\hline A R T I C L E I N F O & A B S T R A C T \\
\hline Review Article & Bacillus Calmette-Guérin (BCG) vaccine against pulmonary tuberculosis (TB) \\
Vackes, 2019 & exhibits poor protective efficacy. However, BCG is the only licensed vaccine \\
Vol. 6, No.1, $29-32$ & against human TB. This review discusses the main research progress in the field \\
Received: January 16, 2020 & main challenges for the development of a safer and more efficient TB vaccine. \\
Accepted: March 11, 2020 &
\end{tabular}

*Corresponding Author: Patricia

Méndez-Samperio, $\mathrm{MD}, \mathrm{PhD}$

Departamento de Inmunología

Escuela Nacional de Ciencias

Biológicas, IPN. Prol. Carpio y Plan

de Ayala. CDMX 11340 México

Email:

pmendezsamperio@gmail.com

Tel/Fax: 5557296000ext62499

KEYWORDS: BCG, Global Health,

Clinical Trials, Tuberculosis, Vaccine

\section{INTRODUCTION}

Currently, the global tuberculosis (TB) epidemic is a public health emergency. TB has been one of the most important diseases in the human history caused by Mycobacterium tuberculosis (M. tb) [1]. M. tb was discovered in 1882 by Robert Koch and is responsible for more deaths than any other human pathogen [2-4]. Beijing and Haarlem genotypes of $M . t b$ are the prevalent genotypes responsible for multidrug resistant TB. Two meetings, the G20 Leader's Declaration (June 2017) [5] and Stop TB Partnership Board Meeting in Delhi (March 2018) chose TB as the highest priority over other infectious diseases. According to recent data by World Health Organization (WHO), an estimated 1.7 million deaths are due to TB each year [6]. Mycobacterium bovis bacillus Calmette-Guérin (BCG) is a live attenuated strain of M. bovis that is used as an anti-tuberculosis vaccine in many countries. BCG vaccination was developed between 1908 and 1921 by Albert Calmette and Camille Guérín in France. BCG was first administered to humans in 1921 and has been used for more than 98 years until now. In 1931, Calmette reported his research of three decades in which established that BCG was truly attenuated. Interestingly, BCG vaccination at birth is associated with decreased mortality in infants [7, 8]. Currently, BCG vaccination is widely practiced around the world and is the most widely used vaccine in human history with more than four billion doses given $[9,10]$. To date, there have been several explanations for the poor protection of the BCG vaccine against pulmonary $\mathrm{TB}$, such as the loss of many genetic regions encoding important immunodominant antigens [11, 12]. However, none of the explanation has clearly defined the observed variations in the efficacy of BCG against pulmonary $\mathrm{TB}$, the most common form of the disease responsible for transmission of the bacterium. In addition, protection against BCG decreases after approximately 20 years [13]. Currently, in the absence of another alternative, BCG remains the only licensed vaccine against human $\mathrm{TB}$ and continues to be used in the immunization programs of different countries [14]. Therefore, development of new $\mathrm{TB}$ vaccines with the understanding for providing better degree of protection than the current BCG vaccine represents an important public health priority.

\section{Inactivated Whole-Cell Mycobacterial Vaccines}

Recent advances in the TB vaccine development include many different approaches. An important TB vaccine approach in clinical trials includes the inactivated whole-cell mycobacterial vaccines, namely Mycobacterium vaccae (M. vaccae) vaccine, VPM1002 vaccine and Mycobacterium indicus pranii (M. indicus pranii; MIP) vaccine [15-17]. Important advantages of this vaccine approach are the overexpression of multistage immunodominant antigens and their safety usage in patients with HIV infection [18-20]. In addition, it has been demonstrated that mucosal vaccination 
with whole-cell TB vaccines provides a better degree of vaccine protective efficacy against $M$. $t b$ than parenteral vaccination $[21,22]$

\section{Live-Attenuated TB Vaccines to Replace BCG}

Recent vaccination approaches to develop better vaccines against human TB include live attenuated vaccines. The current status of the development of live vaccine candidates designed to replace licensed BCG vaccines is progressing [23-25]. To date, MTBVAC and VPM1002 vaccines are two important candidates in the clinical $\mathrm{TB}$ vaccine pipeline, intending to replace BCG. MTBVAC is a vaccine which is rationally attenuated from a clinical $M . t b$ strain and is developed by Universidad de Zaragoza (Spain) and Institut Pasteur (France) with the support of the Tuberculosis Vaccine Initiative [26]. Later clinical development was done by Biofabri Company (Spain) [27]. Interestingly, this TB vaccine has many genetic regions encoding important immunodominant antigens absent in BCG [28]. In addition, this vaccine has demonstrated improved protection compared to BCG in adults and new-born animal models [29-31]. It is important to consider that in clinical phase 1 , this vaccine demonstrated comparable safety to BCG [32]. VPM1002 vaccine is a recombinant BCG developed by the Max Planck Institute in Berlin in collaboration with Vakzine Projekt Management (Germany), and the support of Serum Institute of India and the Tuberculosis Vaccine Initiative. This TB vaccine has been genetically manipulated to permit its escape from the endosome, resulting in cytosolic antigen processing [33-35]. In preclinical studies, VPM1002 vaccine has demonstrated significant protective efficacy [3639]. Currently, studies on this vaccine are underway in South Africa to evaluate it in efficacy trials aimed to prevent TB in HIV-exposed and non-HIV-exposed new-borns [40]. Importantly, this $\mathrm{TB}$ vaccine may improve $\mathrm{BCG}$ vaccine protective efficacy for use in both infants and adults.

\section{BCG Vaccine Protective Efficacy}

Vaccine efficacy refers to the percentage decrease of TB in a vaccinated group of persons compared to an unvaccinated group. Controversies surrounding the protective efficacy of BCG vaccine account for variations in BCG vaccination policies. While BCG vaccine is believed to provide a significant protection against severe forms of TB (i.e. military and meningeal) during the childhood, most adult persons remain susceptible to pulmonary $\mathrm{TB}$ despite $\mathrm{BCG}$ vaccination [41]. Although the BCG vaccine's protective efficacy is questionable, BCG vaccine is considered safe in immunocompetent individuals [42]. However, being a live vaccine, it can result in serious illnesses in immunocompromised patients [43]. In fact, individuals with primary immunodeficiency diseases are in a great risk of complications and represent a challenging group regarding BCG vaccination [44]. Therefore, BCG vaccination should be avoided for the immunodeficient patients [45].

The limited vaccine efficacy of BCG vaccine contributes to the inability of current programs to control TB adequately [46]. In order to improve BCG efficacy against pulmonary TB in current clinical TB vaccine pipelines, BCG boosting strategies and development of vaccines as a replacement for BCG, are being considered [47]. Twenty one years has passed since the quest for a more effective vaccine against human TB. Here, I would like to address main challenges, difficulties and future proposals in this regard. These issues may help to identify key areas which need attention to confer improved safety and protection compared to current BCG vaccine, in the future years. To enhance the vaccine's protective efficacy which can regulate the immune responses during vaccination against TB, future research priorities should be focused on the delivery systems including diversification of the vaccine's administration routes, immunization order, immunization times and immunization intervals. In particular, to induce significant immunity at the site of infection for the maximal effectiveness, the present challenge is to determine a method of administration for vaccines against TB. Moreover, it would be crucial to choose the antigen delivery platform that is best capable of inducing an advanced level of protection over BCG. Currently, intradermal vaccination is the route by which BCG in clinical trials is administered. However, this method of BCG administration does not reliably protect against pulmonary TB. To overcome this issue, inhalation appears to be a better route of the vaccine delivery than intradermal vaccination. This is due to the fact that an inhaled vaccine mimics the route of initial $M$. $t b$ infection. In this context, mucosal vaccination against TB by intranasal or aerosol administration induce increased protection by prompting strong regional tissue immunity. However, BCG vaccination administered via the aerosol route induces significant local immune responses in the lungs with a weak circulating immunity. This important gap can be overcome in the future scientific investigations by the combined use of intradermal with aerosol administrations in order to induce both strong regional tissue and circulating immunities. In this regard, it is important to consider that big challenges to make an aerosol TB vaccine include formulation, selection of the aerosolizing device and to determine whether mucosal vaccination by aerosol administration is useful for human TB vaccination. Therefore, the future vaccine research is necessary to amplify aerosol vaccination studies in endemic areas for TB to determine the safety and efficacy of this method of administration. Currently, it is well known that intravenous administration of BCG to rhesus macaques has elicited superior protection against airborne TB. Therefore, the future strategic directions might include this method of administration when contemplating vaccination against human TB and it could also be considered to diversify the route of the vaccine administration to induce a longer duration of protective immunity than BCG. A big challenge in this issue is to find an optimal dose to induce significant immune responses in the lung. In this regard, dose-scaling studies in TB-endemic countries to conserve a strong immunological memory of $M$. $t b$ can be designed in the future studies.

Another critical challenge is the importance of distinguishing the populations who are potentially sensitive in studying the efficacy of a BCG-replacement vaccine. In this context, the most sensitive population is the healthy neonates who have no pre-existing immunity to their environmental mycobacteria. In addition, adolescents and adults are important target population as the pulmonary forms are responsible for the transmission of the disease. However, a decline in BCG protection with an increase in age is well known. It is important to consider that in this older target population, vaccination with a BCG-replacement vaccine could result in a significant masking effect, due to pre-existing immune responses resulting from exposition to a variety of mycobacterial challenges which may inhibit the protection elicited by BCG. Interestingly, a current disadvantage in TB vaccine candidates based on liveattenuated $M . t b$ is that their administration in 
immunocompromised individuals could be dangerous. To overcome this issue in the future investigations, a combination of different deletions into the same $M$. $t b$ strain could be used to improve the deletion of $M$. $t b$ virulence genes.

\section{Immune Mechanisms Necessary to Improve BCG Vaccine Protective Efficacy}

An important strategy for TB vaccine development is diversification of the immune mechanisms that contribute to the vaccine's protective efficacy, including unrestricted $T$ cells, antibodies, and B lymphocytes. Such studies in clinical trials will allow enhancing the correlation between the immune responses and the protective efficacy of TB vaccine candidates against human TB. In this regard, the challenge of achieving a BCG-replacement vaccine is to understand the adequate immunological mechanisms to protect against $M$. $t b$ infection and disease. In this context, it is critical to recognize the vaccine-specific biomarkers needed to acquire protective efficacy in clinical trials and to confirm the protective immune mechanisms in clinical efficacy studies. To date, no information has been reported for BCG-replacement vaccines in terms of confirmation of the protective immunological mechanisms in clinical efficacy studies. Thus, further scientific investigations are needed to identify the relevant vaccine-specific biomarkers to obtain protective efficacy data in the clinical trials. In particular, future studies should address the efficacy and safety targets in the clinical development strategies. This would be required to develop a novel replacement vaccine to BCG, licensed for the prevention of $M$. $t b$ infection and progression to TB disease in countries with high incidences of TB. In this regard, computerized tools must be considered to correlate the immune protection against TB in humans. In particular, the application of computational technologies utilizing three to four transcripts to identify signatures of the vaccine's safety and efficacy could be very useful.

\section{CONCLUSION}

Research on human TB vaccination has made tremendous progress over the last 21 years as evidenced by M. vaccae, VPM1002 and MIP vaccines which have advanced into phase 3 of the clinical trials. Indeed, at least 12 novel TB vaccine candidates are now in different phases of clinical trials, as reported in January 2018 by Aeras (USA). However, in order to reduce the TB epidemic in high-incidence countries, there is still much to do for development of vaccines against human TB; particularly, vaccines with improved safety and protective efficacy over BCG. Therefore, the challenge for human TB vaccination continues. Currently, TB costs the global economy approximately US $\$ 21$ billion annually [48]. Therefore, an effective TB vaccine represents a critically important strategic goal for controlling the TB epidemic. Although global funding for research in neglected infectious diseases in 2017 was the highest amount ever recorded, the investments in human TB research has remained stable [49]. Since vaccination is the single most cost-effective method to control human TB, additional infusion of funds should be mobilised to support the development of a more efficient vaccine against human TB.

\section{ACKNOWLEDGEMENT}

The author is a researcher of COFAA, EDI, and SNI.

\section{CONFLICT OF INTEREST}

The author declares that she has no conflict of interest.

\section{REFERENCES}

1. Jagielski T, Minias A, van Ingen J, Rastogi N, Brzostek A, Zaczek A et al. Methodological and clinical aspects of the molecular epidemiology of Mycobacterium tuberculosis and other mycobacteria. Clin Microbiol Rev. 2016; 29: (2):239-90. doi: 10.1128/CMR.00055-15.

2. Ottenhoff THM. Overcoming the Global Crisis: "yes, we can," but also for TB? Eur. J Immunol. 2009; 39 (8): 2014-20. doi.org/10.1002/eji.200939518

3. Kaufmann SHE, Winau F. From Bacteriology to Immunology: The Dualism of Specificity. Nat Immunol. 2005; 6(11):1063-6.

4. Kaufmann SH, Hussey G, Lambert PH. New vaccines for tuberculosis. Lancet 2010; 375(9731):2110-9. doi: 10.1016/S0140-6736(10)60393-5.

5. G20 Leader's Declaration. Shaping an Interconnected World Hamburg. (2017). Available from: https://www.g20germany.de/Content/EN/ Anlagen/G20/ G20-leadersdeclaration.pdf;jsessionid=F0BA55C873138F2879E05D2AD6D-

7BA9E.s6t1? blob=publicationFile \&v $=11$

6. WHO. Global Tuberculosis Report 2018. Geneva: World Health Organization; 2018. Licence: CC BY-NC-SA 3.0 IGO.

7. Higgins JP, Soares-Weiser K, Lopez-Lopez JA et al. Association of BCG, $\mathrm{DTP}$, and measles containing vaccines with childhood mortality: systematic review. BMJ 2016; 355:i5170. doi: 10.1136/bmj.i5170

8. Pollard AJ, Finn A, Curtis N. Non-specific effects of vaccines: plausible and potentially important, but implications uncertain. Arch Dis Child. 2017;102(11):1077-1081. doi: 10.1136/archdischild-2015-310282.

9. McShane, H. Tuberculosis Vaccines: Beyond Bacille Calmette-Guerin. Philos. Trans R Soc Lond B Biol Sci. 2011; 366 (1579): 2782-9. doi.org/10.1098/rstb.2011.0097

10. Ottenhoff THM, Kaufmann SHE. Vaccines against Tuberculosis: Where Are We and Where Do We Need to Go? PLoS Pathog. 2012; 8(5): e1002607-12.

doi.org/10.1371/journal.ppat.1002607

11. Nguipdop-Djomo P, Heldal E, Rodrigues LC, Abubakar I, Mangtani P. BCG vaccination: a long lasting protection against tuberculosis?-

Authors' reply. Lancet Infect Dis. 2016;16(4):408-9. doi: 10.1016/S14733099(16)00134-1.

12. Méndez-Samperio P. Current challenges and opportunities for bacillus Calmette-Guérin replacement vaccine candidates. Scand J Immunol. 2019;90(4):e12772. doi: 10.1111/sji.12772.

13. Mangtani P, Nguipdop-Djomo P, Keogh RH et al. Observational study to estimate the changes in the effectiveness of bacillus Calmette-Guérin (BCG) vaccination with time since vaccination for preventing tuberculosis in the UK. Health Technol Assess. 2017; 21(39):1-54. doi: $10.3310 /$ hta 21390 .

14. World Health Organization: BCG vaccine: WHO position paper, February 2018 - Recommendations. Vaccine. 2018; 36 (24): 3408-10. doi.org/10.1016/j.vaccine.2018.03.0090264-410X/Ó2018

15. Yang X, Chen Q, Cui X, Yu Y, Li Y. Mycobacterium vaccae vaccine to prevent tuberculosis in high risk people: a meta-analysis. J Infect. 2010;60 (5):320-30. doi:10.1016/j.jinf.2010.02.005

16. Gupta A, Ahmad FJ, Ahmad F et al. Efficacy of Mycobacterium indicus pranii immunotherapy as an adjunct to chemotherapy for tuberculosis and underlying immune responses in the lung. PloS ONE. 2012; 7(7):e39215. doi: 10.1371 journal.pone.0039215

17. Montangnani C, Chiappini E, Galli L, de Martino M. Vaccine against tuberculosis: what's new? BMC Infect Dis. 2014;14 Suppl 1:S2. doi: 10.1186/1471-2334-14-S1-S2

18. Scriba TJ, Kaufmann SH, Henri Lambert P, Sanicas M, Martin C, Neyrolles O. Vaccination against tuberculosis with whole-cell mycobacterial vaccines. J Infect Dis. 2016; 214(5):659-64. doi: 10.1093/infdis/jiw228.

19. Prabowo SA, Groschel MI, Schmidt E et al. Targeting multidrug resistant tuberculosis (MDR-TB) by therapeutic vaccines. Med Microbiol Immunol. 2013; 202(2):95-104. doi: 10.1007/s00430-012-0278-6.

20. Huang CY, Hsieh WY. Efficacy of Mycobacterium vaccae immunotherapy for patients with tuberculosis: a systematic review and meta-analysis. Hum Vaccin Immunother. 2017;13 (9):1960-71. doi: 10.1080/21645515.2017.1335374

21. Perdomo C, Zedler U, Kuhl AA et al. Mucosal BCG vaccination induces protective lung-resident memory $\mathrm{T}$ cell populations against tuberculosis. MBio. 2016; 7(6). pii: e01686-16. doi: 10.1128/mBio.01686 16.

22. Aguilo N, Alvarez-Arguedas S, Uranga S et al. Pulmonary but not subcutaneous delivery of BCG vaccine confers protection to tuberculosissusceptible mice by an interleukin 17-dependent mechanism. J Infect Dis. 
2016; 213(5):831-9. doi: 10.1093/infdis/jiv503.

23. Kaufmann SHE, Lange C, Rao M et al. Progress in tuberculosis vaccine development and host-directed therapies - a state of the art review. Lancet Respir Med. 2014;2(4):301-20. doi.org/10.1016/S2213-2600(14)70033-5

24. Kaufmann SH, Evans TG, Hanekom WA. Tuberculosis vaccines: time for a global strategy. Sci Transl Med. 2015; 7(276):276fs8. doi: $10.1126 /$ scitranslmed.aaa4730.

25. Méndez-Samperio P. Global efforts in the development of vaccines for tuberculosis: requirements for improved vaccines against Mycobacterium tuberculosis. Scand J Immunol. 2016; 84(4):204-10. doi: 10.1111/sji.12465

26. Arbues A, Aguilo JI, Gonzalo-Asensio J et al. Construction, characterization and preclinical evaluation of MTBVAC, the first live attenuated M. tuberculosis-based vaccine to enter clinical trials.

Vaccine. 2013;31(42):4867-73. doi.org/10.1016/j.vaccine.2013.07.051

27. Walker KB, Brennan MJ, Ho MM et al. The second Geneva consensus: recommendations for novel live TB vaccines. Vaccine. 2010;28(11):225970. doi:10.1016/j.vaccine.2009.12.083

28. Gonzalo-Asensio J, Marinova D, Martin C, Aguilo N. MTBVAC: attenuating the human pathogen of tuberculosis (TB) toward a promising vaccine against the TB epidemic. Front Immunol. 2017; 8:1803. doi: 10.3389/fimmu.2017.01803.

29. Aguilo N, Gonzalo-Asensio J, Alvarez-Arguedas S et al. Reactogenicity to major tuberculosis antigens absent in BCG is linked to improved protection against Mycobacterium tuberculosis.

Nat Commun. 2017; 8:16085. doi: 10.1038/ncomms16085.

30. Clark S, Lanni F, Marinova D, Rayner E, Martin C, Williams A. Revaccination of Guinea pigs with the live attenuated Mycobacterium tuberculosis vaccine MTBVAC improves BCG's protection against tuberculosis. J Infect Dis. 2017; 216(5):525-533. doi: 10.1093/infdis/jix030. 31. Aguilo N, Uranga S, Marinova D, Monzon M, Badiola J, Martin C. MTBVAC vaccine is safe, immunogenic and confers protective efficacy against Mycobacterium tuberculosis in newborn mice. Tuberculosis (Edinb). 2016 ;96:71-4. doi: 10.1016/j.tube.2015.10.010.

32. Spertini F, Audran R, Chakour R et al. Safety of human immunisation with a live-attenuated Mycobacterium tuberculosis vaccine: a randomised, doubleblind, controlled phase I trial. Lancet Respir Med. 2015; 3(12):953 62. doi: 10.1016/S2213-2600(15)00435-X.

33. Grode L, Ganoza CA, Brohm C, Weiner III J, Eisele B, Kaufmann SH. Safety and immunogenicity of the recombinant BCG vaccine VPM1002 in a phase 1 open label randomized clinical trial. Vaccine. $2013 ; 31(9): 1340-8$. doi: 10.1016/j.vaccine.2012.12.053

34. Kaufmann SHE, Cotton MF, Eisele B et al. The BCG replacement vaccine VPM1002: from drawing board to clinical trial. Expert Rev Vaccines. 2014; 13(5):619-30. doi: 10.1586/14760584.2014.905746. 35. Saiga H, Nieuwenhuizen N, Gengenbacher $\mathrm{M}$ et al. The recombinant BCG DeltaureC::hly vaccine targets the AIM2 inflammasome to induce autophagy and inflammation. J Infect Dis. 2015; 211(11):1831-41. doi: 10.1093/infdis/jiu675

36. Grode L, Seiler P, Baumann S et al. Increased vaccine efficacy against tuberculosis of recombinant Mycobacterium bovis bacille Calmette-Guerin mutants that secrete listeriolysin. J Clin Invest. 2005; 115(9):2472-9. 37. Vogelzang A, Perdomo C, Zedler U et al. Central memory CD4+ T cells are responsible for the recombinant bacillus Calmette-Guerin DeltaureC:hly vaccine's superior protection against tuberculosis. J Infect Dis. 2014; 210(12):1928-37. doi: 10.1093/infdis/jiu347.

38. Gengenbacher M, Nieuwenhuizen N, Vogelzang A et al. Deletion of nuoG from the vaccine candidate Mycobacterium bovis BCG DeltaureC:hly improves protection against tuberculosis. MBio. 2016; 7(3). pii: e00679-16. doi: $10.1128 / \mathrm{mBio} .00679-16$.

39. Gengenbacher M, Kaiser P, Schuerer S, Lazar D, Kaufmann SH. Postexposure vaccination with the vaccine candidate bacillus CalmetteGuerin DeltaureC:hly induces superior protection in a mouse model of subclinical tuberculosis. Microbes Infect. 2016; 18(5):364-8. doi: 10.1016/j.micinf.2016.03.005.

40. Nieuwenhuizen NE, Kulkarni PS, Shaligram U et al. The Recombinant Bacille Calmette-Guérin vaccine VPM1002: Ready for clinical efficacy testing. Front Immunol. 2017; ;8:1147. doi: 10.3389/fimmu.2017.01147.

41. Dockrell HM, Smith SG. What have we learnt about BCG vaccination in the last 20 years? Front Immunol. 2017; 8:1134. doi: 10.3389/fimmu.2017.01134.

42. World Health Organization. Meeting of the Global Advisory Committee on Vaccine Safety, June 2017. (2017) July, 2017. Report No.: Contract No. 28.

43. Bonilla FA. Update: vaccines in primary immunodeficiency. J Allergy Clin Immunol. 2018; 141(2):474-481. doi: 10.1016/j.jaci.2017.12.980.

44. Marciano BE, Huang CY, Joshi G, Rezaei N, Carvalho BC, Allwood Z et al. BCG vaccination in patients with severe combined immunodeficiency: complications, risks, and vaccination policies. J Allergy Clin Immunol (2014) 133(4):1134-41. doi:10.1016/j.jaci.2014.02.028.

45. Medical Advisory Committee of the Immune Deficiency Foundation, Shearer WT, Fleisher TA, Buckley RH, Ballas Z, Ballow M et al. Recommendations for live viral and bacterial vaccines in immunodeficient patients and their close contacts. J Allergy Clin Immunol. 2014; 133(4):961-6. doi: 10.1016/j.jaci.2013.11.043.

46. Méndez-Samperio P. Development of tuberculosis vaccines in clinical trials: Current status. Scand J Immunol. 2018;88: 88(4):e12710. doi: 10.1111/sji.12710.

47. Global clinical portfolio of TB vaccine candidates | Aeras. www.aeras.org/pages/global-portfolio. Accessed January 8, 2018.

48. Stop TB Partnership. Global report on tuberculosis vaccines 2018; 2018 [cited 2018 Nov 15]. Available from: http://www.stoptb.org/assets/documents/about/cb/meetings/30/30$09 \% 20$ The $\% 20$ urgent $\% 20$ need $\% 20$ for $\% 20$ global $\% 20$ investment $\% 20 \mathrm{in} \% 20$ TB\%20vaccines $\% 20$ to $\% 20$ eradicate $\% 20$ TB/30-9.1\%20ExecSummary\%20\%20Global\%20Report\%20on\%20Tuberculosis\%20Vaccines\%202018.pdf 49. Cures $\quad$ Research. $\quad 2018$. https://www.policycuresresearch.org/g-finder-2018 (accessed Feb 26, 2019) 